\title{
Enterocolitis Neutropénica en pacientes pediátricos con Leucemia Linfoide Aguda con recaída o refractariedad de su enfermedad de base, durante el período 2016-2017.
}

*Correspondencia:

andres_gonzalezc@hotmail.com

Teléfono [593] 985901559

Conflicto de intereses: Los autores declaran no tener conflictos de intereses.

Fondos: Ver la página 18

Recibido: 30 Diciembre 2017

Aceptado: 11 Marzo 2018

Publicado: 30 Abril 2018

Membrete bibliográfico:

Gonzalez A, Valarezo G, Alvarado $D$, Jiménez $F$, Bonilla A, Espín L. Enterocolitis neutropénica en pacientes pediátricos con leucemia linfoide aguda con recaída o refractariedad de su enfermedad de base, durante el período 2016-2017. Rev. Oncol. Ecu 2018;28(1):9-21.

DOI: https://doi.org/10.33821/257

Copyright Gonzalez, et al. Este artículo es distribuido bajo los términos de Creative Commons Attribution License, el cual permite el uso y redistribución citando la fuente y al autor original.

\section{Neutropenic enterocolitis in pediatric patients with acute lymphoid leukemia with relapse or refractoriness of their underlying disease, during the period 2016-2017.}

\section{Andrés Gonzalez Cabrera ${ }^{\text {* }}$ (iD, Gilda Valarezo Jiménez1, Diana Alvarado Soto1, Fernando Jiménez Torres', Aníbal Bonilla Núñez1, Luis Espín Custodio1.}

1. Instituto Oncológico Nacional "Dr. Juan Tanca Marengo", Solca-Guayaquil, Servicio de Oncología Pediátrica.

\section{Resumen}

Introducción: La enterocolitis neutropénica se define como un proceso inflamatorio multifactorial propio de pacientes neutropénicos, caracterizado por el daño trasmural principalmente a nivel del ciego, aunque puede afectar otras áreas como el colon ascendente e íleo. Debido a la dificultad en el diagnóstico y las complicaciones que se presentan se decide realizar un estudio descriptivo de esta patología, sus métodos diagnósticos, manejo y principales complicaciones asociadas.

Métodos: Entre octubre del año 2016 y septiembre del año 2017, se realizó un estudio retrospectivo y descriptivo de pacientes diagnosticados de Leucemia Linfoide Aguda (LLA) con recaída o refractariedad de su enfermedad de base que presentaron cuadros compatibles clínica y radiográficamente con enterocolitis neutropénica. El estudio fue realizado en el servicio de oncología pediátrica del Instituto Oncológico Nacional "Dr. Juan Tanca Marengo", Solca Guayaquil.

Resultados: Se identificaron 21 casos, de los cuales 17 (80.95\%) fueron hombres. Los principales síntomas presentados fueron dolor abdominal, diarrea y fiebre, en el contexto de pacientes neutropénicos, quienes habían recibido tratamiento quimioterápico en los días previos. La ecografía abdominal fue utilizada en todos los casos junto con tomografía abdominal realizada en 6 pacientes 
como método complementario al diagnóstico. Ningún paciente fue intervenido quirúrgicamente. Todos los pacientes se manejaron de forma conservadora con una evolución favorable.

Conclusión: Debe sospecharse esta patología en todo paciente que presente sintomatología compatible con dolor abdominal, asociando fiebre o diarrea y que hubiere recibido tratamiento quimoterápico en los días previos. Solicitando de forma urgente los estudios complementarios necesarios para el diagnóstico y debiendo ser manejado de forma integral. Reservándose el tratamiento quirúrgico ante cuadros con una evolución desfavorable.

Palabras Claves: ENTEROCOLITIS NEUTROPÉNICA, LEUCEMIA, CÁNCER, DOLOR ABDOMINAL.

DOI: $10.33821 / 257$

\section{Abstract}

Introduction: Neutropenic enterocolitis is defined as a multifactorial inflammatory process typical of neutropenic patients, characterized by transmural damage mainly at the level of the cecum, although it can affect other areas such as the ascending colon and ileus. Due to the difficulty in the diagnosis and the complications that are presented, a descriptive study of this pathology, its diagnostic methods, management and main associated complications is carried out.

Methods: Between October 2016 and September 2017, a retrospective and descriptive study of patients diagnosed with Acute Lymphoblastic Leukemia (ALL) with relapse or refractoriness of their underlying disease who presented clinically and radiographically compatible conditions with neutropenic enterocolitis was performed. The study was carried out in the pediatric oncology service of the National Oncological Institute "Dr. Juan Tanca Marengo ", Solca Guayaquil.

Results: A total of 21 cases were identified, 17 (80.95\%) of which were male. The main symptoms presented were abdominal pain, diarrhea and fever, in the context of neutropenic patients, who had received chemotherapy in the previous days. Abdominal ultrasound was used in all cases together with abdominal tomography performed in 6 patients as a complementary method to the diagnosis. No patient was operated on. All patients were managed conservatively with a favorable evolution.

Conclusion: This pathology should be suspected in any patient who presents symptoms compatible with abdominal pain, associated with fever or diarrhea and who had received chemotherapy in the previous days. The complementary studies necessary for the diagnosis must be requested urgently, and the patient must be managed in an integral manner. Reserving the surgical treatment for patients with an unfavorable evolution.

Keywords: NEUTROPENIC ENTEROCOLITIS, LEUKEMIA, CANCER, ABDOMINAL PAIN.

DOI: $10.33821 / 257$

\section{Introducción}

La Enterocolitis Neutropénica (EN) también conocida como tiflitis, se define como un proceso inflamatorio multifactorial, propio de los pacientes neutropénicos que se caracteriza por el daño trasmural, principalmente a nivel del ciego aunque puede afectar otras áreas como el colon ascendente e íleo [1, 2]. La patogénesis no se encuentra totalmente identificada sin embargo se considera que el uso de agentes quimioterápicos y 
el daño a la mucosa intestinal, junto con la propia neutropenia estarían implicados, lo que predispondría al desarrollo de procesos infecciosos $[3,4]$.

En la actualidad es amplio el número de fármacos implicados y aunque algunos de los primeros agentes relacionados fueron los taxanos hoy destaca de forma importante la citarabina e idarrubicina, no obstante con el empleo de nuevos regímenes que utilizan altas dosis de quimioterápicos se espera que la incidencia de otros fármacos también se incremente [5-8]. Sin embargo la incidencia real de esta patología es desconocida, la mayoría los estudios la sitúan en un $5.3 \%$ aproximadamente, con una mayor frecuencia en las neoplasias hematológicas, principalmente las leucemias agudas y linfomas [9-10]. Observándose en pacientes pediátricos una incidencia mayor (11.6\%) [11-12].

La EN se presenta como un cuadro caracterizado un síndrome doloroso abdominal en el contexto de un paciente neutropénico, sin embargo suelen sumarse otros síntomas como fiebre, diarrea, distensión, vómitos, defensa abdominal, hemorragia digestiva baja, e inclusive signos de choque que se puede evidenciar en cuadros más avanzados [12, 13]. Destacando que el desarrollo de los mismos coincide en ocasiones con una caída brusca en la neutropenia y aumentos en las cifras de proteína $C$ reactiva, usualmente entre los días 7 a 10 de iniciado el tratamiento $[14,15]$.

Con respecto al diagnóstico además de la clínica, el uso de técnicas de imagen es indispensable siendo la radiografía de abdomen altamente inespecífica y destacando la ecografía abdominal junto a la tomografía abdominal por su mayor sensibilidad [16, 17]. Así la medición del grosor de la pared intestinal por ecografía ha demostrado ser útil en el diagnóstico y en el seguimiento por su relación con la severidad del cuadro, considerándose es la mayoría de estudios un grosor superior a $3 \mathrm{~mm}$ patológico y relacionando un aumento mayor a $10 \mathrm{~mm}$ con una mayor mortalidad [18-20]. A su vez la tomografía abdominal destaca por su capacidad para diferenciar otras patologías gastrointestinales propias de pacientes neutropénicos [21, 22].

En relación al tratamiento se recomienda que este sea multidisciplinario e individualizado tomando en cuenta los factores de riesgo, e incluye el reposo intestinal si es necesario con nutrición parenteral, el uso de sondaje nasogástrico, el tratamiento antimicrobiano de amplio espectro y además la corrección de citopenias y coagulopatías [23-25]. El tratamiento quirúrgico está indicado en pacientes que presenten cuadros de mayor gravedad como perforación intestinal, íleo, obstrucción intestinal o que no evidencien mejoría a pesar del manejo clínico [26, 27].

Para el manejo antimicrobiano se considera en la mayoría una infección polimicrobiana relacionada a microorganismos gramnegativos y anaerobios, por lo que la mayoría de guías consideran el uso de monoterapia con un carbapenem o con piperacilina-tazobactam o una combinación de una cefalosporina más metronidazol (por su actividad anaeróbica) como regímenes apropiados, incluso como medida profiláctica sin establecer el papel del tratamiento antimicótico [28, 29].

El objetivo de este estudio es identificar la incidencia en el desarrollo de EN en pacientes pediátricos con recaída de su enfermedad de base, sometidos a quimioterapias de rescate en el Servicio de Oncología Pediátrica de Sociedad de Lucha Contra el Cáncer (SOLCA) 
Guayaquil. Debido a la dificultad en el diagnóstico y la importancia del manejo precoz e integral, para evitar el desarrollo de complicaciones potencialmente mortales se decide realizar el siguiente reporte.

\section{Materiales y Métodos}

Se realizó una revisión de todos los expedientes clínicos de pacientes pediátricos diagnosticados de leucemia linfoide aguda con recaída o refractariedad de su enfermedad de base, que coincidiendo con el desarrollo de neutropenia tras tratamiento quimioterápico, presentaron cuados clínicos y hallazgos radiológicos compatibles con EN, durante el periodo comprendido entre octubre del año 2016 y septiembre del año 2017 en el Servicio de Oncología Pediátrica del Instituto Oncológico Nacional "Dr. Juan Tanca Marengo", Solca-Guayaquil. Se excluyeron del estudio aquellos casos con expediente clínico incompleto.

Se estudiaron las variables relacionadas a sexo, edad, fase del tratamiento, quimioterapia recidiva, tiempo en que se desarrolló el cuadro clínico, estudios diagnósticos realizados, tratamiento recibido, complicaciones y posterior evolución. La información se obtuvo del registro interno del hospital y se recolectó en una base de datos para el posterior análisis estadístico. Se realiza el reporte bajo la supervisión de la jefatura del servicio.

\section{Resultados}

Durante el periodo de estudio se identificó un total 21 casos de EN, de los cuales 17 (80.95 $\%$ ) correspondieron a varones y el resto a mujeres, presentado una media de 8 años de edad. El inicio de los síntomas se observó en promedio tras $8.5( \pm 2.96)$ días de comenzado el tratamiento quimioterápico, presentándose en la mayor parte de los casos antes del décimo día ( $n=15,74.43 \%)$. La mayoría de los pacientes $(n=20,95.24 \%)$ presentaron un recuento absoluto de neutrófilos menor a 500 células $/ \mathrm{mm} 3$ al inicio de los síntomas; con una media de $6 \pm 4.22$ días de neutropenia profunda previos. El único paciente con un contaje superior presentó una caída súbita de valor total de neutrófilos 48 horas después. Los paciente prensaron además en el $57.14 \%$ de los casos una cifra de proteína $C$ reactiva (PCR) mayor a mg/dl al momento del diagnóstico (Tabla 1). Entre los principales agentes quimioterápicos recibidos se encontraban la citarabina e idarrubicina 19 (90.48\%), el etopósido, la ciclofosfamida y los corticoides 18 (85.71\%) (Tabla 2).

Los principales síntomas presentados fueron en 17 (80.95\%) dolor abdominal, en 15 (71.43 \%) diarrea, en 11 (52.38\%) fiebre y en 7 (33.33\%) tanto náuseas y/o vómitos como distensión; cabe destacar 2 pacientes (9.52 \%) que presentaban un cuadro de mucositis de evolución tórpida al inicio de los síntomas, y 1 paciente (4.76 \%) que presentó un cuadro de shock séptico que requirió manejo en el área de cuidados intensivos pediátricos (Tabla 3). 
Tabla 1: Características principales de los pacientes con Enterocolitis Neutropénica

\begin{tabular}{|c|c|c|}
\hline VARIABLE & N. & $\%( \pm \mathrm{DS})$ \\
\hline \multicolumn{3}{|c|}{ Sexo } \\
\hline MASCULINO & 17 & 80.95 \\
\hline FEMENINO & 4 & 19.05 \\
\hline \multicolumn{3}{|c|}{ Media de Edad en Años } \\
\hline & 8 & $( \pm 3.5)$ \\
\hline \multicolumn{3}{|c|}{ Media de días de Inicio de los Síntomas } \\
\hline & 8.5 & $( \pm 2.96)$ \\
\hline \multicolumn{3}{|c|}{ RAN (células/mm3) } \\
\hline$>500$ & 1 & 4.76 \\
\hline $500-100$ & 4 & 19.05 \\
\hline$<100$ & 16 & 76.19 \\
\hline \multicolumn{3}{|c|}{ Cifra de PCR $(\mathrm{mg} / \mathrm{dl})$} \\
\hline$>5$ & 12 & 57.14 \\
\hline $5-1$ & 2 & 9.52 \\
\hline$<1$ & 5 & 23.81 \\
\hline \multicolumn{3}{|c|}{ Media de días de Neutropenia Previos } \\
\hline & 6 & $( \pm 4.22)$ \\
\hline \multicolumn{3}{|c|}{ Media de días de duración del cuadro } \\
\hline 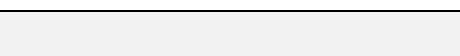 & 11.57 & $( \pm 3.25)$ \\
\hline
\end{tabular}

DS: desviación estándar. RAN: recuento absoluto de neutrófilos. PCR: proteína C reactiva

Tabla 2. Principales agentes quimioterápicos utilizados

\begin{tabular}{|l|c|c|}
\hline Fármaco & $\mathrm{N}$. & $\%$ \\
\hline Citarabina & 19 & 90.48 \\
\hline Etopósido & 18 & 85.71 \\
\hline Ciclofosfamida & 18 & 85.71 \\
\hline Idarrubicina & 19 & 90.48 \\
\hline Fludarabina & 2 & 9.52 \\
\hline Clofarabina & 1 & 4.76 \\
\hline Vincristina & 2 & 9.52 \\
\hline Bortezomib & 1 & 4.76 \\
\hline Dexametasona & 18 & 85.71 \\
\hline
\end{tabular}

La ecografía abdominal fue utilizada como método complementario al diagnóstico en la totalidad de los casos, la medición del grosor de pared intestinal mostro una media de 0.32 $\pm 0.22 \mathrm{~cm}$. La tomografía abdominal fue realizada en 6 pacientes $(28.57 \%)$ con difícil diagnóstico inicial, evidenciado datos sugestivos de tiflitis en todos ellos, con un grosor de pared intestinal promedio de $0.97 \pm 0.5 \mathrm{~cm}$. La radiografía simple de abdomen se realizó en 12 pacientes presentado la mayoría de ellos $(n=9,75 \%)$ hallazgos inespecíficos (Figuras 1 y 2). 
Tabla 3. Principales signos y síntomas de los pacientes con Enterocolitis Neutropénica.

\begin{tabular}{|l|c|c|}
\hline Signos y Síntomas & N. & $\%$ \\
\hline Dolor & 17 & 80.95 \\
\hline Diarrea & 15 & 71.43 \\
\hline Fiebre & 11 & 52.38 \\
\hline Distensión & 7 & 33.33 \\
\hline Nausea y Vómito & 7 & 33.33 \\
\hline Defensa Abdominal & 3 & 14.29 \\
\hline Mucositis & 2 & 9.52 \\
\hline Shock & 1 & 4.76 \\
\hline Hemorragia Digestiva Baja & 0 & 0.00 \\
\hline
\end{tabular}

Se reportaron un total de 15 hemocultivos, de los cuales 9 (60\%) no presentaron crecimiento bacteriano tras 5 días, el principal germen aislado fue el bacilo Gram negativo E. coli que se halló en el $26.67 \%(n=4)$ de los hemocultivos reportados.

La totalidad de los pacientes fueron manejados de manera conservadora con cobertura antibiótica de amplio espectro destacando el uso de piperacilina-tazobactam en el 80.95 $\%$ de los casos $(n=17)$, reposo intestinal, nutrición por vía parenteral en todos los pacientes y transfusiones de acuerdo a las necesidades de hemoderivados. Un paciente requirió de ingreso en la unidad de cuidados intensivos pediátricos por cuadro de sepsis grave asociado a EN. Ningún paciente fue intervenido quirúrgicamente. Todos presentaron una evolución favorable con una media de $11.57 \pm 3.25$ días hasta la recuperación de su cuadro.

Figura 1. Imagen de ecografía abdominal mostrando distención de asas intestinales a nivel de fosa ilíaca izquierda.

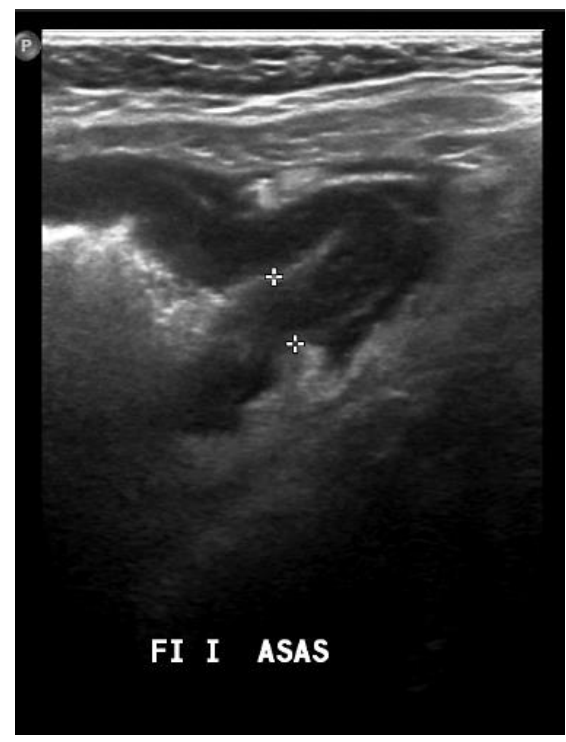


Figura 2. Imagen de tomografía abdominal contrastada en mostrando importante engrosamiento concéntrico de las paredes del colon.

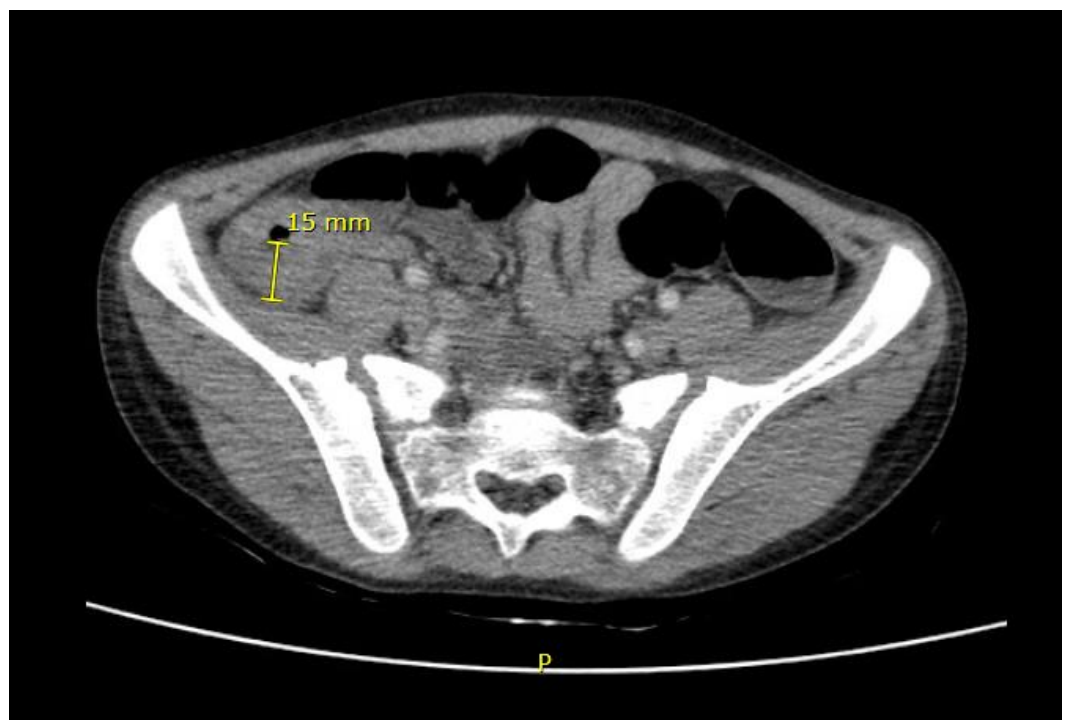

\section{Discusión}

Una de las principales dificultades con respecto a la valoración de la EN es que su incidencia real es aún desconocida, la mayoría de estudios la sitúan en un $5.3 \%$, con mayor frecuencia en las neoplasias hematológicas $[9,10]$, sin embargo algunas series evidencian una incidencia mayor cunado se evalúa a pacientes pediátricos (11.6\%) [12].

Otra de las grandes complicaciones es que su patogénesis no se encuentra totalmente esclarecida, aunque se conoce que múltiples factores estarían implicados entre ellos los mismos agentes quimioterápicos entre los que destacan la citarabina, idarrubicina, etopósido, metotrexato por su efecto de daño directo sobre la pared intestinal provocando áreas ulceradas y necróticas, la vincristina por el desarrollo de íleo junto con distención de la pared abdominal o los esteroides al promover las infecciones sean estas bacterianas 0 fúngicas [6-8], además de la misma neutropenia que predispondría al desarrollo de procesos infecciosos e inclusive sépticos $[1,3,4]$.

La sintomatología se caracteriza por el dolor abdominal que suele localizarse a nivel de fosa iliaca derecha junto con fiebre, diarrea, náuseas, vómitos o distensión abdominal [11, $13,16]$. En el contexto de un paciente que se encuentra atravesando una neutropenia profunda (menor a 500 células $/ \mathrm{mm}^{3}$ ) en muchos casos conjuntamente con anemia y trombocitopenia, similar a lo encontrado en la presente serie. Advirtiéndose además que el desarrollo del cuadro coincide con la caída en las cifras de neutrófilos totales y el aumento de reactantes de fase aguda inespecíficos como la PCR, habitualmente entre los 7 a 10 días de iniciado el tratamiento quimioterápico $[14,15]$.

En relación al diagnóstico, este se basa fundamentalmente en la clínica en con conjunto criterios radiológicos de sospecha, ya que estos últimos no siempre permiten un diagnóstico definitivo [16]. Entre los métodos más comúnmente usados se encuentra la radiografía simple de abdomen, la misma que sin embargo es altamente inespecífica y en 
donde la mayoría de hallazgos se relacionan con presencia de aire libre, asas dilatadas en intestino delgado y borramiento de la línea del psoas derecho [17].

A su vez la ecografía abdominal junto a la tomografía de abdomen destacan por su mayor sensibilidad y especificidad $[17,18]$. Así como por la capacidad para realizar una medición del grosor de la pared intestinal, dato que ha demostrado ser útil en el diagnóstico, seguimiento y pronostico del cuadro, considerando la mayoría de estudios un grosor superior a $3 \mathrm{~mm}$ altamente sugestivo y relacionando un aumento mayor a $10 \mathrm{~mm}$ con una mayor mortalidad $[19,20]$.

Entre otros hallazgos en la ecografía abdominal se observa principalmente paredes intestinales engrosadas, líquido libre en cavidad además de signos sugestivos de edema o necrosis, y esta permite además valorar el grado de vascularización de la pared gracias uso de doppler color, que en la mayoría de los casos refleja hipervascularidad de la mucosa y de la pared intestinal $[17,18]$.

La tomografía abdominal destaca por su capacidad para diferenciar otras patologías relacionadas con síndrome doloroso abdominal en pacientes inmunocomprometidos, y es usada en muchas ocasiones como técnica confirmatoria o en aquellos casos en los que la ecografía no es concluyente [21, 23].

El área más comúnmente afectada es el ciego y colon ascendente esto probablemente relacionado a una menor perfusión de los tejidos, aunque el daño puede encontrarse en cualquier otra parte del intestino delgado o colon ${ }^{2}$. El uso de colonoscopia y enema barritado se encuentra contraindicado ya que puede ocasionar perforación de una pared ya afecta por los cambios antes mencionados [26].

Una gran variedad de microrganismos han sido aliados en hemocultivos, destacando el hallazgo de bacteriemia por bacilos Gram negativos (E. coli), cocos Gram positivos (Enterococcus) y en menor cantidad anaerobios $u$ hongos del genero Cándida [1, 2, 4]. Similar a lo encontrado en el presente estudio en el cual E. coli fue la bacteria más observada en hemocultivos.

Es importante además tomar en cuenta los múltiples diagnósticos diferenciales que se pueden establecer y que suponen un desafío para el manejo y la toma de decisiones como: cuadro apendicular agudo, gastroenteritis de origen bacteriano o viral, colitis pseudomembranosa o isquémica, enfermedad inflamatoria del colon, obstrucción intestinal, o la misma infiltración del intestino por células leucémicas, entre otros [21, 22].

El tratamiento supone un tema de discusión, se sugiere sea multidisciplinario e individualizado tomando en cuenta cada uno de los factores de riesgo, ya que puede variar desde un tratamiento de tipo conservador hasta la necesidad e intervención quirúrgica temprana $[23,24]$.

Como parte del manejo conservador se incluye el reposo intestinal con uso de nutrición parenteral si es necesario, sondaje nasogástrico como medida para la descompresión gástrica, correcto manejo de líquidos y electrólitos, tratamiento antimicrobiano de amplio espectro (con el uso de monoterapia con un carbapenem o con piperacilina-tazobactam o una combinación de una cefalosporina tercera o cuarta generación asociada a metronidazol) o antifúngico si la evolución del paciente así lo requiere y además la corrección de citopenias y coagulopatías. Siendo este el tratamiento de elección en la mayoría de casos siempre que sea iniciado en forma precoz [25-27]. 
La decisión de tratamiento quirúrgico es compleja por el estado en que se encuentran estos pacientes y sus múltiples factores de riesgo. Sin embargo la misma se indica en cuadros que evidencien mayor gravedad entre los que se incluyen: perforación intestinal, persistencia de sangrado gastrointestinal tras la corrección de las citopenias y los trastornos de la coagulación, o desarrollo de abdomen agudo en pacientes sin respuesta al tratamiento conservador. El tipo de técnica utilizada dependerá de la gravedad del cada caso y debe ser individualizada, en el presente estudio no se precisó de este tipo de intervención $[24,26]$.

Los avances en el manejo y el tratamiento de estos pacientes han permitido aumentar la sobrevida de los mismos, sin embargo se considera que existen factores de mal pronóstico entre los que se incluyen la neutropénica prolongada, el desarrollo de cuadros sépticos, el uso de drogas vasopresoras y un grosor intestinal mayor de $10 \mathrm{~mm}$, por su relación con una mayor duración de los síntomas y de la mortalidad [16, 18, 19]. A su vez factores como una corta duración de la neutropenia en relación al uso de estimulantes de colonias de granulocitos (G-CSF) o la presentación del cuadro con síntomas más discretos pueden considerarse de buen pronóstico; lo que provoca que la mortalidad relacionada a este cuadro sea muy variable, en la presente serie sin embargo todos los pacientes sobrevivieron sin presentar complicaciones posteriores [20, 23, 27].

\section{Conclusiones}

Debe sospecharse de un cuadro de EN frente a todo paciente que presente sintomatología compatible con dolor abdominal, asociando fiebre, diarrea o hemorragia digestiva y que hubiere recibido tratamiento quimioterápico en los días previos. Realizándose de forma urgente estudios diagnósticos complementarios como la ecografía abdominal y la tomografía de abdomen por su mayor sensibilidad y especificidad, con el objetivo de iniciar de forma inmediata el tratamiento más adecuado para cada paciente en función de su evolución.

Los pacientes deben ser manejados de forma integral, recomendándose además el uso de hemocultivos, coprocultivos y otras pruebas necesarias para la detención de gérmenes causales y su tratamiento especificado. El tratamiento quirúrgico es una alternativa frente a cuadros complicados o con una mala evolución, y la decisión con respecto al tipo de intervención a realizar será conforme a cada paciente.

\section{Agradecimientos}

Reconocemos a las personas que participaron indirectamente en el estudio tales como el personal técnico, pacientes y personal del Instituto Oncológico Nacional "Dr. Juan Tanca Marengo", Solca-Guayaquil. 


\section{Información adicional}

Nota del Editor

La Revista Oncología Ecu permanece neutral con respecto a los reclamos jurisdiccionales en mapas publicados y afiliaciones institucionales.
Abreviaturas

EN: Enterocolitis Neutropénica.

G-CSF: estimulantes de colonias de granulocitos.

Archivos Adicionales

Ninguno declarado por los autores.

Fondos

Los fondos de la investigación fueron propios de los autores del presente artículo.

\section{Disponibilidad de datos y materiales}

Existe la disponibilidad de datos bajo solicitud al autor de correspondencia. No se reportan otros materiales.

\section{Contribuciones de los autores}

AGC, GVJ, realizaron la idea de investigación, revisión bibliográfica. DAS, FJT, ABN, LEC, recolección de datos, escritura del artículo. LEC realizó el análisis crítico del artículo. AGC, realizó las correcciones editoriales. Todos los autores leyeron y aprobaron la versión final del artículo.

Aprobación de ética y consentimiento para participar

No aplica a este estudio.

\section{Consentimiento para publicación}

No aplica. 
Andrés Gonzalez Cabrera, Médico Residente del Servicio de Oncología Pediátrica, Instituto Oncológico Nacional "Dr. Juan Tanca Marengo", Solca-Guayaquil. https://orcid.org/0000-0002-4289-3957

Gilda Valarezo Jiménez, Médico Residente del Servicio de Oncología Pediátrica, Instituto Oncológico Nacional "Dr. Juan Tanca Marengo", Solca-Guayaquil.

Diana Alvarado Soto, Médico Residente del Servicio de Oncología Pediátrica, Instituto Oncológico Nacional “Dr. Juan Tanca Marengo”, Solca-Guayaquil.

Fernando Jiménez Torres, Médico Residente del Servicio de Oncología Pediátrica, Instituto Oncológico Nacional "Dr. Juan Tanca Marengo", Solca-Guayaquil.

Aníbal Bonilla Núñez, Médico Tratante del Servicio de Oncología Pediátrica, Instituto Oncológico Nacional "Dr. Juan Tanca Marengo", Solca-Guayaquil.

Luis Espín Custodio, Jefe de Servicio de Oncología Pediátrica, Instituto Oncológico Nacional "Dr. Juan Tanca Marengo", Solca-Guayaquil.

Revisiones por pares

Acceda a la revisión de pares académicos en el siguiente enlace: https://publons.com/review/3830522

Abreviaturas en la referencias DOI: Digital Object Identifier PMID: PubMed Identifier SU: Short URL

\section{Referencias}

1. Rodrigues F, Da Silva G, Wexner S. Neutropenic enterocolitis. World J Gastroenterol. 2017;23:42-47. DOI: 10.1097/PAS.0000000000000517

2. Sachak T, Arnold M, Naini B, Graham R, Shah S, Cruise M, et al. Neutropenic Enterocolitis. Am J Surg Pathol. 2015;39(12):1635-42. DOI: 10.1097/PAS.0000000000000517

3. Davila M. Neutropenic enterocolitis. Curr Opin Gastroenterol. 2006;22. PMID: 16319675

4. Ullery B, Pieracci F, Rodney J, Barie P. Neutropenic enterocolitis. Surg Infect (Larchmt). 2009;10(3):30714. DOI: $\underline{10.1089 / \text { sur.2008.061 }}$

5. Pugliese N, Salvatore $P$, lula D, Catania M, Chiurazzi F,Della R. Ultrasonography-driven combination antibiotic therapy with tigecycline significantly increases survival among patients with neutropenic enterocolitis following cytarabine-containing chemotherapy for the remission induction of acute myeloid leukemia. Cancer Med. 2017;6(7):1500-1511. DOI: 10.1002/cam4.1063

6. Camera A, Andretta C, Villa M, Volpicelli M, Picardi M, Rossi M, et al. Intestinal toxicity during induction chemotherapy with cytarabine-based regimens in adult acute myeloid leukemia. Hematol J. 2003;4(5):346-50. DOI: 10.1038/sj.thj.6200304

7. Hogan WJ, Letendre L, Litzow M, Farhadfar N, Cerquozzi S, Hessenauer M, et al. Neutropenic colitis after treatment of acute myelogenous leukemia with idarubicin and cytosine arabinoside. Mayo Clin Proc. 2002;77(8):760-2. DOI: $\underline{10.1080 / 10428194.2016 .1222379}$ 
8. Vlasveld L, Zwaan F, Fibbe W, Tijon, Tham P. Neutropenic enterocolitis following treatment with cytosine arabinoside-containing regimens for hematological malignancies: a potentiating role for amsacrine. Ann Hematol. 1991;62(4):129-34. SU: goo.gl/yFGc8H

9. Gorschlüter M1, Marklein G, Höfling K, Clarenbach R, Baumgartner S, Hahn C, et al. Abdominal infections in patients with acute leukaemia: a prospective study applying ultrasonography and microbiology. $\mathrm{Br} \mathrm{J}$ Haematol. 2002;117(2):351-8. PMID: 11972517

10. Gorschlüter M1, Mey U, Strehl J, Ziske C, Schepke M, Schmidt-Wolf I, et al. Neutropenic enterocolitis in adults: systematic analysis of evidence quality. Eur J Haematol. 2005;75(1):1-13. DOI: 10.1111/j.1600$\underline{0609.2005 .00442 . x}$

11. Shafey A, Ethier MC, Traubici J, Naqvi A, Sung L. Incidence, risk factors, and outcomes of enteritis, typhlitis, and colitis in children with acute leukemia. J Pediatr Hematol Oncol. 2013;35(7):514-7. DOI: 10.3748/wjg.v23.i1.42

12. Mullassery D, Bader A, Battersby A, Mohammad Z, Jones L, Parmar C, et al. Diagnosis, incidence, and outcomes of suspected typhlitis in oncology patients--experience in a tertiary pediatric surgical center in the United Kingdom. J Pediatr Surg. 2009;44(2):381-5.

13. Portugal R, Nucci M. Typhlitis (neutropenic enterocolitis) in patients with acute leukemia: a review. Expert Rev Hematol. 2017;10(2):169-174. DOI: 10.1016/i.jpedsurg.2008.10.094

14. Fike F, Mortellaro V, Juang D, St peter S, Andrews W, Snyder L. Neutropenic colitis in children. J Surg Res. 2011;170(1):73-6. DOI: 10.1016/j.jss.2011.01.041

15. Machado NO. Neutropenic enterocolitis: A continuing medical and surgical challenge. N Am J Med Sci. 2010;2(7):293-300. PMID: 22558577

16. Mccarville MB, Adelman CS, Li C, Furman WL, Razzouk BI, Pui CH, et al. Typhlitis in childhood cancer. Cancer. 2005;104(2):380-7. DOI: 10.1002/cncr.21134

17. Sloas MM, Flynn PM, Kaste SC, Patrick CC. Typhlitis in children with cancer: a 30-year experience. Clin Infect Dis. 1993;17(3):484-90. PMID: 8218694

18. Dietrich CF, Hermann S, Klein S, Braden B. Sonographic signs of neutropenic enterocolitis. World J Gastroenterol. 2006;12(9):1397-402. DOI: 10.3748/wjg.v12.i9.1397

19. Rizzatti M, Brandalise SR, de Azevedo AC, Pinheiro VR, Aguiar Sdos S. Neutropenic enterocolitis in children and young adults with cancer: prognostic value of clinical and image findings. Pediatr Hematol Oncol. 2010;27(6):462-70.

20. Cartoni C, Dragoni F, Micozzi A, Pescarmona E, Mecarocci S, Chirletti P, et al. Neutropenic enterocolitis in patients with acute leukemia: prognostic significance of bowel wall thickening detected by ultrasonography. J Clin Oncol. 2001;19(3):756-61. DOI: 10.1200/JCO.2001.19.3.756

21. Kirkpatrick ID, Greenberg HM. Gastrointestinal complications in the neutropenic patient: characterization and differentiation with abdominal CT. Radiology. 2003;226(3):668-74. DOI: $\underline{10.1148 / \text { radiol.2263011932 }}$

22. Spencer SP, Power N, Reznek RH. Multidetector computed tomography of the acute abdomen in the immunocompromised host: a pictorial review. Curr Probl Diagn Radiol. 2009;38(4):145-55. DOI: 10.1067/i.cpradiol.2007.11.001

23. Sullivan P, Moreno C. A Multidisciplinary Approach to Perianal and Intra-Abdominal Infections in the Neutropenic Cancer Patient. Oncology (Williston Park, NY). 2015;29(8):581-90. PMID: 26281844

24. Sundell $\mathrm{N}$, Boström $\mathrm{H}$, Edenholm $\mathrm{M}$, Abrahamsson $\mathrm{J}$. Management of neutropenic enterocolitis in children with cancer. Acta Paediatr. 2012;101(3):308-12. DOI: 10.1111/j.1651-2227.2011.02465.x

25. Freifeld AG, Bow EJ, Sepkowitz KA, et al. Clinical practice guideline for the use of antimicrobial agents in neutropenic patients with cancer: 2010 update by the infectious diseases society of america. Clin Infect Dis. 2011;52(4):e56-93. DOI: $10.1093 / \mathrm{cid} / \mathrm{cir073}$ 
26. Schnell D, Azoulay E, Benoit D, Boeckh MJ, Ito JI, Mullen CA, et al. Management of neutropenic patients in the intensive care unit (NEWBORNS EXCLUDED) recommendations from an expert panel from the French Intensive Care Society (SRLF) with the French Group for Pediatric Intensive Care Emergencies (GFRUP), the French Society of Anesthesia and Intensive Care (SFAR), the French Society of Hematology (SFH), the French Society for Hospital Hygiene (SF2H), and the French Infectious Diseases Society (SPILF). Ann Intensive Care. 2016;6(1):90. DOI: 10.1093/cid/cir073

27. Nesher L, Rolston KV. Neutropenic enterocolitis, a growing concern in the era of widespread use of aggressive chemotherapy. Clin Infect Dis. 2013;56(5):711-7.

28. Gafter-gvili A, Fraser A, Paul M, Leibovici L. Meta-analysis: antibiotic prophylaxis reduces mortality in neutropenic patients. Ann Intern Med. 2005;142(12 Pt 1):979-95. PMID: 15968013

29. Gorschlüter M, Mey U, Strehl J, Volker S, Christian R, Katharina $\mathrm{P}$, et al. Invasive fungal infections in neutropenic enterocolitis: a systematic analysis of pathogens, incidence, treatment and mortality in adult patients. BMC Infect Dis. 2006;6:35. DOI: 10.1186/1471-2334-6-35 